Bistua:Revista de la Facultad de Ciencias Básicas.2016.14(2):03-14

\title{
DOSIMETRÍA IN VIVO EN TRATAMIENTOS DE CANCER DE CÉRVIX CON Ir-192
}

\author{
Gustavo Alvarino $\mathrm{B}^{1}$, Enrique Pardo $\mathrm{P}^{2}$, Alfonso Quijano $\mathrm{P}^{3}$
}

${ }^{1}$ Grupo de Física Médica.Departamento de Física. Universidad de Córdoba. Montería, Colombia.

${ }^{2}$ Grupo de Investigación Genes.Departamento de Biología. Universidad de Córdoba.Montería, Colombia.

${ }^{3}$ Grupo de Investigación en Química. Laboratorio de Control de Calidad. Universidad de Pamplona. Colombia

\section{Resumen}

Para asegurar la calidad en los tratamientos de braquiterapia de alta tasa de dosis intracavitarios en Ca de cérvix, es necesario que los fotones de Ir - 192 entreguen la dosis prescrita, con la mayor precisión posible, en el tumor y se debe minimizar la distribución de dosis, en el tejido sano y órganos críticos que rodean al tumor, tan baja como sea razonablemente deseable. Por esta razón debemos hacer la medición directamente sobre el tejido humano. En este trabajo se realiza el proceso de medición en pacientes con cáncer de cérvix tratados con Ir -192, en los cuales utilizamos dosímetros TLD-100 de LiF: Mg; Ti, que se ubican en recto y vejiga para comprobar la dosis real recibida, utilizando la dosimetría in vivo, comparándola con la medición arrojada por el sistema de planeación Abacus 3.1, puesto que estos órganos deben recibir menos del $70 \%$.

Palabras clave: Braquiterapia HDR, Dosimetría in vivo, Termoluminiscencia

\section{DOSIMETRY IN VIVO IN CERVICAL CANCER TREATMENT WITH Ir-192}

\section{Abstract}

To assure the quality in the treatments of Braquiterapia of discharge rate of dose intracavitarios in cervix, it is necessary that the photons of $\mathrm{Ir}-192$ give the dose prescribed with the biggest possible precision in the tumor and the dose distribution should be minimized in the healthy fabric and critical organs that surround to the tumor, as low as it is reasonably desirable, it is for it that we should make it directly on human fabric. In this work he/she is carried out a reception of patient with cervix cancer tried with Ir-192, in which we 


\section{4}

use dosímetros TLD-100 of LiF: Mg; Ti that are located in right and bladder to check the received real dose using the dosimetría in alive, with which throws the planeacion system Abacus 3.1, since these organs should receive less than $70 \%$ of the dose prescribed to the tumor in the cervix.

Keywords: HDR Brachytherapy, in vivo dosimetry, thermoluminescence

*Para citar este artículo: Alvarino B.G, Pardo P.E, Quijano Parra A- Dosimetria in vivo en tratamientos de cáncer de cérvix con Ir-192. Revista Bistua. 2016.14(2):03-14

+ Autor para el envió de correspondencia y la solicitud de las separatas: Alvarino B.G. Grupo de Física Médica.Departamento de Física. Universidad de Córdoba.Colombia, e-mail: alvarinog@gmail.com 
05

\section{Introducción}

En el presente trabajo, desarrollado en el Instituto de Cancerología de Sucre (INCAS) ubicado en la ciudad de Sincelejo en el Departamento de Sucre, República de Colombia, mediante dosimetría in vivo, se estiman las dosis suministradas a pacientes en tratamientos de braquiterapia. Esta herramienta permite estimar la precisión del tratamiento prescrito, la detección de errores sistemáticos y la prevención de accidentes radiactivos; contribuyendo a la evaluación de incertidumbres en la planeación y aplicación de dosis en un sitio de tratamiento específico.

La braquiterapia es una técnica muy utilizada en tratamientos de cáncer, usando principalmente haces de fotones (1). Los implantes intracavitarios e intersticiales de las fuentes se realizan por medio de aplicadores, usando sistemas a control remoto, los cuales permiten ubicarlas dentro del volumen blanco o muy cerca de él.

Aunque las aplicaciones de estos tratamientos, se rigen por protocolos internacionales, emanados de la International Atomic Energy Agency (IAEA), tales como (2) o (3). No existe en Colombia, un programa de control de calidad de dosimetría in vivo, el cual garantice que las dosis calculadas y aplicadas en planeaciones dosimétricas, sean las que realmente estén recibiendo los pacientes.

El (3) recomienda una tolerancia de \pm $5 \%$ como desviación estándar relativa a la precisión de la dosimetría clínica. Para verificar el cumplimiento de este rango, se hace necesario implementar técnicas de dosimetría in vivo.

Este trabajo, pretende estimar las dosis prescritas en los tratamientos, usando detectores TLD-100 (LiF: Mg, Ti) que por su tamaño, en forma de barra, son introducidos en las cavidades naturales del cuerpo humano y por sus propiedades tales como alta precisión, buena estabilidad ambiental y linealidad de las respuestas en el rango de 0 hasta 1000 cGy, elementos de gran importancia en los tratamientos de braquiterapia HDR, permitiendo su uso como dosímetros de radiación.

Adicionalmente, otras características de estos cristales son su densidad: $2,64 \mathrm{~g} \mathrm{/} \mathrm{cm}^{3}$; número atómico efectivo $Z_{\text {ef: }}$ 8.20; número de electrones por gramo: $2.786 \times 10^{23}$, las cuales los hacen compatibles con el tejido del músculo humano. El número atómico efectivo, se calcula usando la ecuación de (4) definido por $Z_{\text {ef }}=\left(a_{1} Z_{1}^{2.94}+\cdots a_{n} Z_{n}^{2.94}\right)^{1 / 2.94}$ Donde $a_{1, \mathrm{~m}} a_{n}$, son las contribuciones fraccionales de los elementos presentes en el material. 
06

\section{Materiales y métodos}

Calibración de los cristales TLD100 con la energía del Co-60 (1.25 $\mathrm{MeV}$ )

Se tomaron los cristales TLD-100 en forma de barras y se calibraron con la energía del Co-60, ubicándolos a 80 $\mathrm{cm}$ de la fuente con un buildup de 0.5 $\mathrm{cm}$ de acrílico para la energía promedio de $1.25 \mathrm{MeV}$ del Co-60 y se irradiaron con una dosis de 50 cGy en un Theratron 780, usando un campo de referencia de $10 \times 10 \mathrm{~cm}^{2}$. El rendimiento en profundidad se verificó con una cámara de ionización de 0,66 CC tipo Farmer, certificada por el Laboratorio Secundario de Calibración dosimétrica de INGEOMINAS.

Para calcular el rendimiento se siguieron las recomendaciones del protocolo de calibración dosimétrico señalado por (5), dado por:

$$
R=M^{*} P_{\cup} S_{W, a r} N_{D, W}
$$

Donde,

$R$ es el rendimiento o tasa de dosis a una profundidad de referencia en agua o material equivalente.

$M^{*}$, es la lectura en Roentgen por división de escala, corregidas por presión y temperatura.

$S w$, air, es la relación entre los poderes de frenado del agua al aire $P_{U}$, es el factor de corrección de perturbación que tiene en cuenta la no equivalencia del material de las paredes de la cámara

$N_{D, w}$ es el factor de calibración de la cámara en cGy por Roentgen por división de escala.

Posteriormente se leyeron los cristales en el equipo Harshaw Modelo 4500, obteniendo la lectura individual de cada uno.

(Li) y la lectura promedio $(\bar{L})$, determinando un factor de sensibilidad de corrección (ki) para cada cristal, dado por:

$$
K_{i}=\frac{L_{i}}{L}
$$

El proceso siguiente fue irradiar con dosis en el rango desde 300cGy hasta 750 cGy, en grupos de cuatro cristales, los cuales fueron leídos, promediando las lecturas obtenidas para cada uno de los grupos de cristales.

Al graficar las dosis D (cGy) entregadas en función de las lecturas $\mathrm{L}(\mu \mathrm{C})$ de la señal $\mathrm{TL}$ y se obtuvo la Figura 1.

Al hacer el ajuste lineal, se obtiene la ecuación

$$
D_{L}(\sigma G y)=0,5579 \sigma G y+0,3164(\sigma G y / \mu C) * L(\mu C)
$$

Donde,

$D_{L}$ : Es la dosis determinada mediante el cálculo.

L: Es la lectura obtenida del cristal en unidades de carga $(\mu C)$ 
07

Estos datos tienen una desviación estándar de 0.338227 y un nivel de confiabilidad de $99.484 \%$.

Esta calibración para la calidad del haz del Co-60 puede ser usada para determinar las energías de otros radionúclidos, siempre y cuando se corrija por el factor que lo lleve a la energía del haz utilizado. La relación de las respuestas de los estos cristales por unidad de dosis (cGy) con el Co-60 para las energías del Cs-137, Ra-226, y rayos X de 2.8 MV es de 1.00 y para el Ir-192 es de 1.04 -1.06 (6).

En este trabajo no hubo necesidad de utilizar este factor porque la calibración con la fuente de Ir-192 se realizó a la misma profundidad y en las mismas condiciones en que se realizan las medidas de dosis.

La calibración de los cristales con la energía del Ir-192, se realizó conectando un catéter endobronquial tipo $6 \mathrm{~F}$ a la unidad HDR y se determinó el posicionamiento de la fuente mediante placa radiográfica. Los cristales se ubicaron a $10 \mathrm{~cm}$ de profundidad desde la fuente, separados por Material JJT (7), sobre el eje transversal de la fuente. Se les administraron dosis entre 300cGy hasta 750 cGy. Los datos obtenidos con el lector Harshaw Modelo 4500, se muestran en la Tabla 2.

Al realizar la gráfica de dosis en función de la lectura se obtuvo la gráfica No.2. Los cuales dan cuenta del comportamiento lineal de las respuestas en el rango de 0 hasta 1000 cGy.

El ajuste lineal que se realizó a los datos experimentales arrojó los siguientes resultados:

$$
Y=A+B X
$$

Donde, $A=9.80636 ; B=13.1763 ; S D$ $=6.1675$; Correlación $R=0.9992$. Por consiguiente la ecuación utilizada para calcular las dosis teniendo en cuenta las lecturas de los cristales es la siguiente:

$$
D_{L}=9.80636 c G y+13.1763 \frac{c G y}{\mu C} * L(\mu C)
$$

Siendo $L(\mu C)$ la lectura arrojada por los cristales irradiados y a los cuales se les quiere determinar las dosis que han recibido. La validez de la curva de calibración fue verificada con un grupo de cristales utilizados para este fin.

\section{Planeación de los Tratamientos}

Al colocar el aplicador en las pacientes, consistente en un anillo de $26 \mathrm{~mm}$ de diámetro y un tándem de $40 \mathrm{~mm}$ ó $60 \mathrm{~mm}$ de longitud, se procede a verificar la posición correcta del sistema por medio de fluoroscopia; tal como se muestra en la Figura 2.

Posteriormente se introducen catéteres por el recto y la vejiga, a 
08

través de los cuales se introducen fuentes ficticias, con el objeto de determinar los puntos de ubicación de los cristales utilizados para medir las dosis, en cuatro puntos de cada una de esas cavidades.

Con este montaje se toman las dos placas: una AP y otra lateral, necesarias para realizar la planeación del tratamiento y obtener las curvas de isodosis.

Generalmente, los tratamientos se prescriben a puntos $A(6)$, aplicando dosis desde 750 hasta 300 cGy. La dosis en las Figuras 3 y 4 es de 600 cGy prescritas a puntos A. Las curvas de isodosis que se obtienen con este sistema tienen la forma que se presenta en las Figuras 4 y 5 .

El Sistema Abacus 3.1, permite calcular las dosis en puntos de recto y vejiga que sean señalados en las placas, los cuales se hacen corresponder con los puntos de ubicación de los cristales.

El algoritmo utilizado por el sistema de planeación, considera la fuente puntual para distancias mayores que $2 \mathrm{~L}$, siendo $\mathrm{L}$ la longitud activa de la fuente (8). La fuente de Ir-192 tiene una longitud activa de $3.5 \mathrm{~mm}$ y los cálculos para este tipo de tratamientos se realizan a distancias superiores a $10 \mathrm{~mm}$, considerando el tamaño de la vagina, el útero, la vejiga y el recto de las pacientes.
En general las dosis medidas con los cristales están afectadas por el factor $\left[\frac{x}{x \pm d x}\right]^{2}$, donde $\mathrm{dx}$ es el valor de la incertidumbre. Se observa que si el cristal se coloca a distancia $\mathrm{x} \pm \mathrm{dx}$, recibirá más o menos dosis que en la posición $\mathrm{x}$, debido a la dependencia de las dosis del factor $1 / \mathrm{r}^{2}$. Este error se incrementa en la medida que el punto de cálculo esté más cerca de la fuente, como consecuencia de los altos gradientes que se presentan en cercanías de la misma. Los puntos los $V_{1}, V_{2}, V_{3}$ y $V_{4}$ corresponden a puntos de vejiga, mientras que $R_{1}, R_{2}$, $\mathrm{R}_{3}$ y $\mathrm{R}_{4}$ corresponden a puntos del recto. De igual forma se planean y calculan las curvas de isodosis para aplicaciones de 600cGy y 550cGy, respectivamente. En esas mismas coordenadas, se ubican los cristales para cada uno de los tratamientos, mediante el uso de las placas fluoroscópicas, colocándolos en las posiciones de las fuentes ficticias, escogidas para tal fin. Los resultados obtenidos se comparan con los del sistema de planeación.

\section{Resultados y Discusión}

Un cálculo del Sistema para el Sistema Abacus 3.1, con una dosis de 650 cGy a puntos A, arroja los resultados observados en la Tabla 3 , en la cual las coordenadas de un punto $\mathrm{x}, \mathrm{y}, \mathrm{z}$ se expresan en $\mathrm{mm}$. Los resultados para las curvas de isodosis para aplicaciones de 600cGy 
09

y 550cGy, respectivamente se muestran en las Tablas 4 y 5 .

En la tabla No.6 se muestran los resultados obtenidos al verificar la validez de la curva de calibración, mediante un grupo de cristales escogidos aleatoriamente, los cuales fueron irradiados con dosis entre 300 cGy y 750 cGy, realizada con el haz de Co-60. $D_{e}$ es la dosis entregada al cristal y $D$ L es la dosis obtenida con la lectura del cristal.

A continuación, se presentan las dosis calculadas mediante el Sistema Abacus 3.1 (Ds) y las dosis obtenidas con los cristales TLD-100 (DTLD) y su correlación respectiva. $\mathrm{La}$ prescripción se hace a puntos A. Para los puntos de recto los resultados se presentan en la Tabla No. 7 y para los puntos de vejiga los resultados se presentan en la Tabla 8.

Estos resultados muestran las dosis que se obtienen al promediar las dosis adquiridas mediante la lectura de los cristales ubicados en los cuatro puntos de recto y en los cuatro puntos de vejiga, en comparación con las dosis obtenidas mediante el cálculo realizado por el sistema de planeación.

Al reutilizar los cristales cambia su sensibilidad por lo que es recomendable recalibrarlos individualmente para cada medición, ya que la sensibilidad varía con el tiempo de irradiación en los fósforos, debido a la migración gradual, a temperatura ambiente de las trampas o centros de atrapamiento de electrones en el cristal.

Los resultados obtenidos en la calibración de los cristales con el haz de Co-60, muestran para el cálculo de dosis en aire, una diferencia máxima de $\pm 1.30 \%$ respecto a la dosis esperada, en el rango entre $0 \mathrm{y}$ 750 cGy, como puede observarse en la tabla No.6. Estos resultados confirman que este proceso es excelente para determinar las dosis en este tipo de tratamientos, siempre y cuando se sigan cuidadosamente el tratamiento térmico recomendado en la literatura consistente en una limpieza (annealing), la irradiación y el ciclo de lectura (7).

La calibración de los cristales con la energía del Ir-192 (Promedio 0.38 $\mathrm{MeV}$ ), realizada con un buildup de 2 $\mathrm{mm}$ de espesor con material JJT y a una distancia fuente- cristal de $10 \mathrm{~cm}$ con dosis de 50 cGy, muestran una diferencia máxima de $\pm 1.86 \%$ entre la dosis entregada al cristal y la dosis calculada con la lectura del mismo. Lo cual indica que este resultado está dentro del rango de incertidumbre (2\%) permitido para este tipo de procedimientos (5).

La comparación de los resultados del Sistema Abacus 3.1 con los de las lecturas de los TLD-100, muestra una diferencia menor al $\pm 4.3 \%$, tanto para los puntos de vejiga como para 
10

los puntos de recto, como se observa en las tablas №.6 y №.7, manteniéndose dentro del rango de error permitido $( \pm 5 \%)$ para este tipo de procedimientos (9).

En la localización de los puntos de cálculo sobre las placas radiográficas, AP y Lateral, donde se ubicarán los cristales se comete un error del orden de las décimas de $\mathrm{mm}$ por lo que puede esperarse una incertidumbre del orden de $\pm 1 \mathrm{~mm}$.

\section{Conclusiones}

Los resultados obtenidos mediante la dosimetría TLD-100 de LiF: Mg, Ti, permiten realizar la comparación con los resultados arrojados por el sistema de planeación Abacus 3.1, utilizado en el Instituto de Cancerología de Sucre. Para este tipo de procedimientos. En otras palabras este proceso es la verificación experimental del software del sistema de planeación

Este procedimiento resulta ser apropiado para determinar las dosis in vivo en los órganos en riesgo para este tipo de tratamientos: vejiga y recto; al permitir verificar que las dosis prescritas se están o nó suministrando correctamente a las pacientes y no se exceden las dosis de tolerancia señaladas para estos órganos (10).
Así mismo este tipo de procedimientos permiten sentar las bases para iniciar en el país un Protocolo de Control de Calidad para esta clase de tratamientos de radioterapia.

\section{Referencias bibliográficas}

1.-Johns $\mathrm{H}$. and Cunningham J. The Physics of Radiology. Tomo I. Charles C. Thomas Publisher. Springfield. Illinois. USA. Fourth Edition; 1983.

2.-Technical Reports Series 277. Absorbed Dose Determination in Photon and Electron Beams. Second Edition. An International Code of Practice. International Atomic Energy Agency. 1997.

3.-ICRU Report 59. Dose and Volume Specification for Reporting Interstitial Therapy. International commission on Radiation and Measurements. ICRU. Publications. Bethesda. USA. 1997.

4.-Mayneord W. The significance of de roentgen (Acta Int. Union Against Cancer). Pp. 2-271.1937.

5.-ARCAL XXX. Aspectos Físicos de la Garantía de Calidad en Radioterapia: de Control de Calidad. Proyecto RLA/6/032. "Mejoramiento de la Calidad en Radioterapia". 1999. 
11

6.-Kapp K.S. Dosimetry of Intracavitary Placements for Uterine and Cervical Carcinoma: Results of Orthogonal Film, TLD, and Assisted Techniques: Radiotherapy Oncology. 1992.

7.-Alvarino G. and Plazas M C. Estudio dosimétrico en braquiterapia de alta tasa de dosis con Ir-192 para tratamientos de carcinoma de cérvix en radioterapia. Tesis de Maestría. pp 44. Universidad Nacional de Colombia. Bogotá 2002

8.-Harshaw BICRON. TLD Thermoluminiscense Dosimetry. Manual del Usuario. Saint-Gobain. Cristal \& Detectors. Wermelskirchen. Germany, 2001.

9.-Attix F. Introduction to Radiological Physics and Radiation Dosimetry. John Wiley and Sons. 1986.

Tabla 1. Lectura de los cristales TLD-100 en función de las dosis suministradas con la energía del Co-60

\begin{tabular}{cr}
\hline Dosis (cGy) & Lectura $L(\mu \mathrm{C})$ \\
\hline 300 & 20.57 \\
350 & 24.85 \\
400 & 27.73 \\
450 & 31.50 \\
500 & 35.80 \\
550 & 39.90 \\
600 & 43.65 \\
650 & 46.28 \\
700 & 48.96 \\
750 & 53.55 \\
\hline
\end{tabular}

Tabla 2. Lectura de los cristales TLD-100 en función de las dosis suministradas con la energía del Ir-192

\begin{tabular}{cc}
\hline Dosis (cGy) & Lectura $\mathrm{L}(\mu \mathrm{C})$ \\
\hline 300 & 21.6 \\
350 & 26.1 \\
400 & 29.4 \\
450 & 33.4 \\
500 & 37.6 \\
550 & 41.5 \\
600 & 45.4 \\
650 & 48.6 \\
700 & 51.9 \\
750 & 55.7 \\
\hline
\end{tabular}

10.-ICRU Report 44. Tissue Substitutes in Radiation Dosimetry and Measurement Publications. Bethesda. USA. 1989. 
12

Tabla 3. Dosis calculadas por el sistema para una dosis a puntos $A$ de 650 cGy con tándems de $6 \mathrm{~cm}$

\begin{tabular}{cccccc}
\hline $\begin{array}{c}\text { Punto } \\
\mathrm{s}\end{array}$ & $\mathrm{X}(\mathrm{mm})$ & $\mathrm{Y}(\mathrm{mm})$ & $\begin{array}{c}\mathrm{Z}(\mathrm{mm} \\
)\end{array}$ & $\mathrm{D}(\%)$ & $\mathrm{D}_{(\mathrm{s})}(\mathrm{Gy})$ \\
\hline $\mathrm{V}_{1}$ & -3.9 & -47.0 & 6.2 & 20.15 & 130.98 \\
$\mathrm{~V}_{2}$ & -4.8 & -48.2 & 0.1 & 18.32 & 119.08 \\
$\mathrm{~V}_{3}$ & -5.9 & -49.6 & -5.0 & 16.70 & 108.55 \\
$\mathrm{~V}_{4}$ & -6.5 & -51.7 & -10.5 & 14.82 & 96.33 \\
$\mathrm{R}_{1}$ & 14.5 & 30.8 & -4.8 & 34.89 & 226.79 \\
$\mathrm{R}_{2}$ & 14.1 & 29.9 & -10.8 & 32.70 & 212.55 \\
$\mathrm{R}_{3}$ & 13.5 & 28.7 & -16.2 & 29.62 & 192.53 \\
$\mathrm{R}_{4}$ & 12.8 & 27.2 & -23.2 & 25.88 & 168.22 \\
\hline
\end{tabular}

Tabla.4. Dosis calculadas por el sistema para una dosis a puntos A de 600 cGy con tándems de $6 \mathrm{~cm}$

\begin{tabular}{cccccc}
\hline $\begin{array}{c}\text { Pun } \\
\text { tos }\end{array}$ & $\mathrm{X}(\mathrm{mm})$ & $\begin{array}{c}\mathrm{Y}(\mathrm{m} \\
\mathrm{m})\end{array}$ & $\begin{array}{c}\mathrm{Z}(\mathrm{m} \\
\mathrm{m})\end{array}$ & $\begin{array}{c}\mathrm{D} \\
(\%)\end{array}$ & $\begin{array}{c}\mathrm{D}_{(\mathrm{S})}( \\
\mathrm{Gy})\end{array}$ \\
\hline $\mathrm{V}_{1}$ & -3.9 & -47.0 & 6.2 & 20.1 & 120.90 \\
$\mathrm{~V}_{2}$ & -4.8 & -48.2 & 0.1 & $\begin{array}{c}18.3 \\
2\end{array}$ & 109.92 \\
$\mathrm{~V}_{3}$ & -5.9 & -49.6 & -5.0 & $\begin{array}{c}16.7 \\
0\end{array}$ & 100.20 \\
$\mathrm{~V}_{4}$ & -6.5 & -51.7 & -10.5 & $\begin{array}{c}14.8 \\
2\end{array}$ & 88.92 \\
$\mathrm{R}_{1}$ & 14.5 & 30.8 & -4.8 & $\begin{array}{c}34.8 \\
9\end{array}$ & 209.34 \\
$\mathrm{R}_{2}$ & 14.1 & 29.9 & -10.8 & $\begin{array}{c}32.7 \\
0\end{array}$ & 196.20 \\
$\mathrm{R}_{3}$ & 13.5 & 28.7 & -16.2 & $\begin{array}{c}29.6 \\
2\end{array}$ & 177.72 \\
$\mathrm{R}_{4}$ & 12.8 & 27.2 & -23.2 & 25.8 & 155.28 \\
& & & & 8 & \\
\hline
\end{tabular}

\begin{tabular}{cccccc}
\hline $\begin{array}{c}\text { Punto } \\
\mathrm{s}\end{array}$ & $\mathrm{X}(\mathrm{mm})$ & $\mathrm{Y}(\mathrm{mm})$ & $\begin{array}{c}\mathrm{Z}(\mathrm{mm} \\
)\end{array}$ & $\mathrm{D}(\%)$ & $\mathrm{D}_{(\mathrm{S})}(\mathrm{Gy})$ \\
\hline $\mathrm{V}_{1}$ & -3.9 & -47.0 & 6.2 & 20.15 & 110.83 \\
$\mathrm{~V}_{2}$ & -4.8 & -48.2 & 0.1 & 18.32 & 100.76 \\
$\mathrm{~V}_{3}$ & -5.9 & -49.6 & -5.0 & 16.70 & 91.85 \\
$\mathrm{~V}_{4}$ & -6.5 & -51.7 & -10.5 & 14.82 & 81.51 \\
$\mathrm{R}_{1}$ & 14.5 & 30.8 & -4.8 & 34.89 & 191.90 \\
$\mathrm{R}_{2}$ & 14.1 & 29.9 & -10.8 & 32.70 & 179.85 \\
$\mathrm{R}_{3}$ & 13.5 & 28.7 & -16.2 & 29.62 & 162.91 \\
$\mathrm{R}_{4}$ & 12.8 & 27.2 & -23.2 & 25.88 & 142.34 \\
\hline
\end{tabular}

Tabla 5. Dosis calculadas por el sistema para una dosis a puntos A de 550 cGy con tándems de $6 \mathrm{~cm}$
Tabla 6. Comparación de las dosis entregadas con el haz de Co-60 y las dosis medidas con los cristales

\begin{tabular}{ccc}
\hline$D_{e}(c G y)$ & $D_{L}(c G y)$ & $D_{L} / D_{e}$ \\
\hline 300 & 303.940 & 1.0130 \\
350 & 346.772 & 0.9907 \\
400 & 396.870 & 0.9921 \\
450 & 452.854 & 1.0060 \\
500 & 504.957 & 1.0090 \\
550 & 553.936 & 1.0070 \\
600 & 597.970 & 0.9966 \\
650 & 642.929 & 0.9891 \\
700 & 708.070 & 1.0110 \\
750 & 759.101 & 1.0120 \\
\hline
\end{tabular}

Tabla 7. Dosis promedio calculadas por el sistema, comparadas con las dosis promedio medidas con los cristales TLD- 100 para puntos de recto.

\begin{tabular}{ccccc}
\hline Dosis a puntos A & $\mathbf{D}_{\mathbf{s}}$ (cGy) & D $_{\text {TLD }}$ (cGy) & D TLD / $_{\mathbf{s}}$ & $\%$ \\
\hline 650 & 200.02 & 191.51 & 0.957 & 4.3 \\
600 & 184.63 & 179.31 & 0.971 & 2.9 \\
550 & 169.24 & 162.89 & 0.962 & 3.8 \\
\hline
\end{tabular}

Tabla 8. Dosis promedio calculadas por el sistema, comparadas con las dosis promedio medidas con cristales TLD- 100 para puntos de vejiga.

\begin{tabular}{|l|l|l|l|l|}
\hline $\begin{array}{l}\text { Dosis a } \\
\text { puntos } \\
\text { A }\end{array}$ & $\mathbf{D}_{\mathbf{s}}$ (cGy) & $\mathbf{D}_{\text {TLD }}$ (cGy) & $\begin{array}{l}\mathbf{D}_{\text {TLD }} / \\
\mathbf{D}_{\mathbf{s}}\end{array}$ & $\%$ \\
\hline 650 & 113.73 & 109.41 & 0.962 & 3.8 \\
\hline 600 & 104.98 & 103.46 & 0.985 & 1.5 \\
\hline 550 & 96.24 & 94.83 & 0.985 & 1.5 \\
\hline
\end{tabular}

Bistua Revista de la Facultad de Ciencias Básicas. Universidad de Pamplona-Colombia.ISSN 0120-4211 
Figura.1. Calibración de los cristales TLD-100 de LiF: Mg; Ti con la energía del Co-60

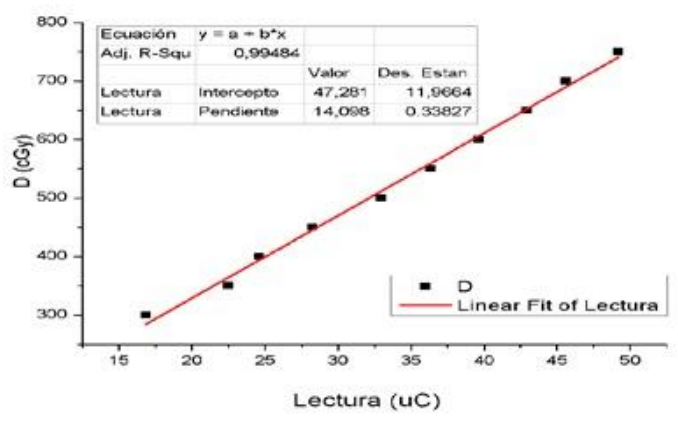

Figura.2.Calibración de los TLD-100 con la energía del Ir-192 (0.38 MeV)
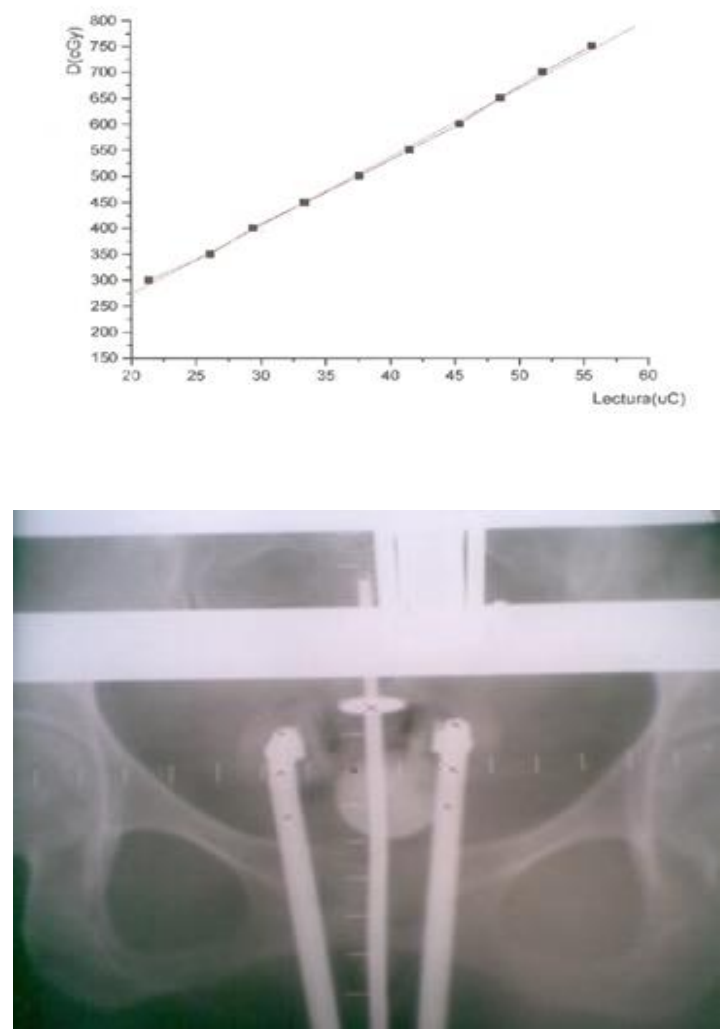

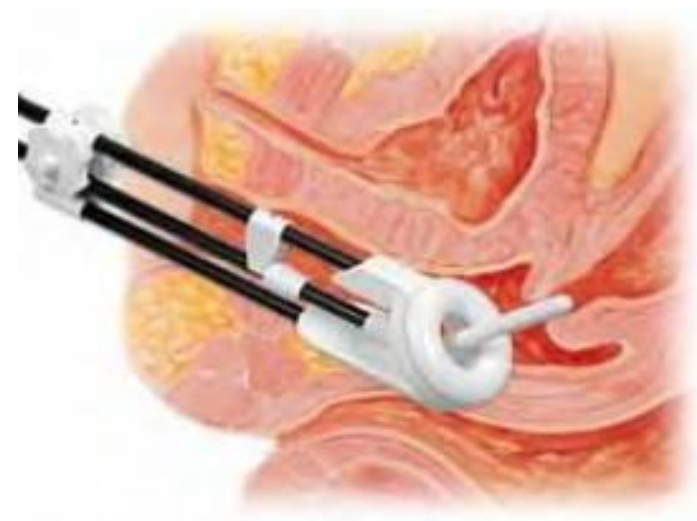

Figura 3. Aplicador utilizado por el Sistema de Planeación Abacus 3.1. El anillo que se adosa al cuello del útero y el tándem penetra en la cavidad uterina. Se observa la vejiga con un medio de contrastes y en la parte inferior el canal rectal (Alvarino, $G$ and Plazas, M, 2002).

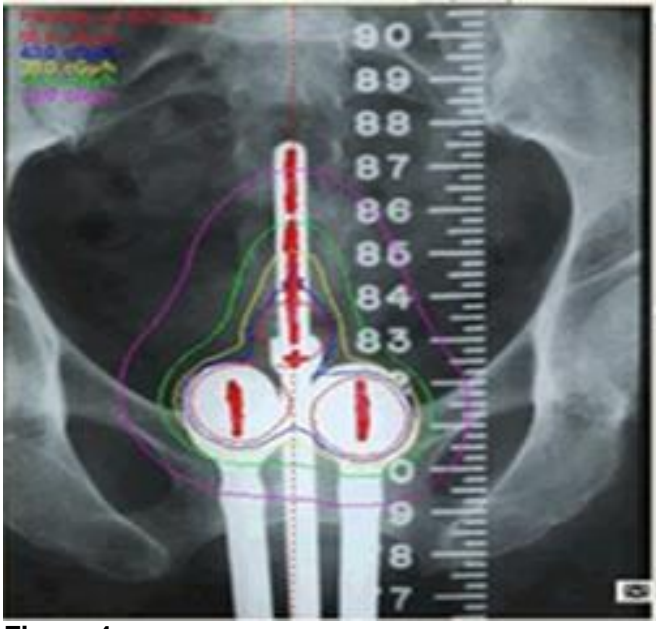

Figura 4

Figura 3 
14

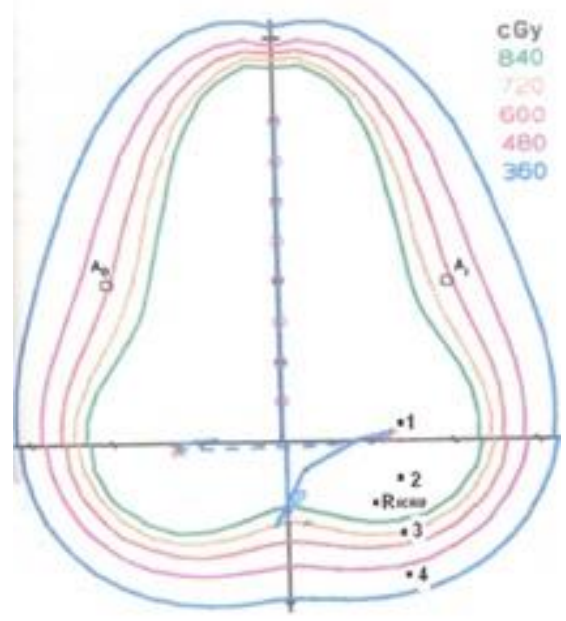

Figura 4. Curvas de isodosis típicas del Sistema Abacus 3.1 (Attix, F, 1986). Se observan los puntos de recto utilizados para ubicación de los cristales.

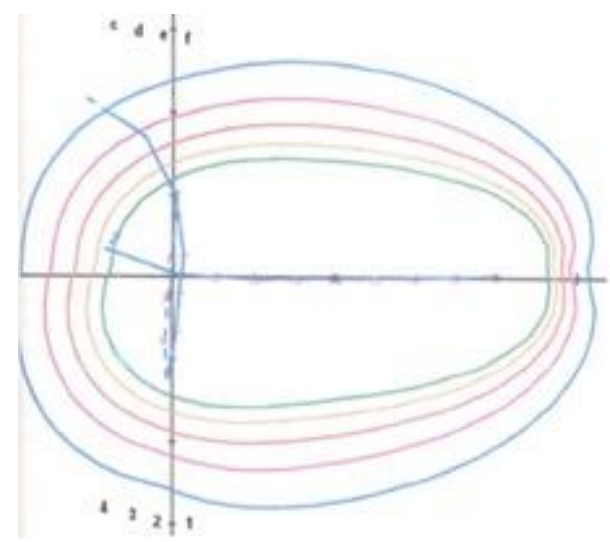

Figura 5. Curvas de isodosis típicas del Sistema Abacus 3.1, en el plano latera I(Attix, $F, 1986)$. Se observan los puntos de vejiga en la parte superior y de recto en la parte inferior, utilizados para ubicación de los cristales.

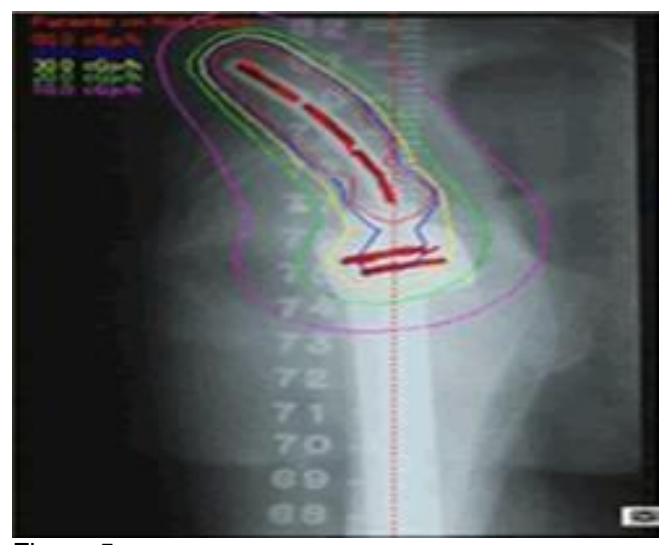

Figura 5 